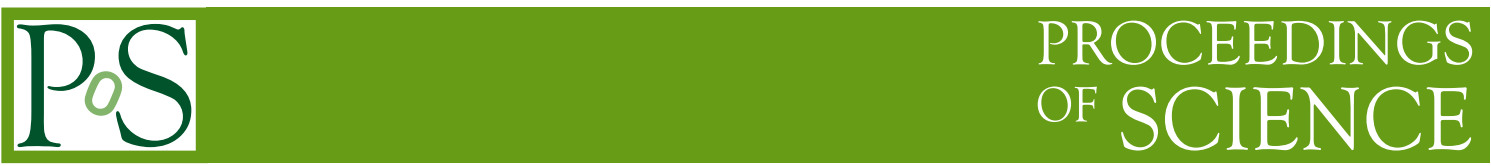

\title{
Renormalization in Tensorial Group Field Theories
}

\author{
Sylvain Carrozza* \\ Centre de Physique Théorique \\ E-mail: sylvain.carrozza@cpt.univ-mrs.fr
}

In this talk, I will review some recent results about the renormalization of Tensorial Group Field Theories. These theories are motivated by an approach to quantum gravity which lies at the crossroad of tensor models and loop quantum gravity. From the mathematical point of view, they are quantum field theories defined on compact Lie groups, with specific non-local interactions. Interestingly, these non-localities can be controlled and several models have now been proven perturbatively well-defined [1, 2, 3, 4, 5, 6]. I will focus on a $S U(2)$ model inspired by Eucliden $3 d$ quantum gravity, which has been proven renormalizable at all orders with up to $\varphi^{6}$ interactions [6]. Time allowing, I will also present new results about the renormalization group flow of this model.

References

[1] J. Ben Geloun and V. Rivasseau, "A Renormalizable 4-Dimensional Tensor Field Theory," Commun. Math. Phys. 318, 69 (2013) [arXiv:1111.4997 [hep-th]].

[2] J. Ben Geloun and D. O. Samary, “3D Tensor Field Theory: Renormalization and One-loop $\beta$-functions,” Annales Henri Poincare 14, 1599 (2013) [arXiv:1201.0176 [hep-th]].

[3] J. Ben Geloun and E. R. Livine, "Some classes of renormalizable tensor models," J. Math. Phys. 54, 082303 (2013) [arXiv:1207.0416 [hep-th]].

[4] S. Carrozza, D. Oriti and V. Rivasseau, "Renormalization of Tensorial Group Field Theories: Abelian $U(1)$ Models in Four Dimensions," Commun. Math. Phys. 327, 603 (2014) [arXiv:1207.6734 [hep-th]].

[5] D. O. Samary and F. Vignes-Tourneret, "Just Renormalizable TGFT's on $U(1)^{d}$ with Gauge Invariance,” Commun. Math. Phys. (2014) [arXiv:1211.2618 [hep-th]].

[6] S. Carrozza, D. Oriti and V. Rivasseau, "Renormalization of an $S U(2)$ Tensorial Group Field Theory in Three Dimensions," Commun. Math. Phys. (2014) [arXiv:1303.6772 [hep-th]].

Frontiers of Fundamental Physics 14 - FFP14,

15-18 July 2014

Aix Marseille University (AMU) Saint-Charles Campus, Marseille

* Speaker. 